Research Article

\title{
Least Square Homotopy Perturbation Method for Ordinary Differential Equations
}

\author{
Mubashir Qayyum (i) and Imbsat Oscar \\ National University of Computer and Emerging Sciences FAST, Lahore, Pakistan \\ Correspondence should be addressed to Mubashir Qayyum; mubashir.qayyum@nu.edu.pk
}

Received 16 July 2021; Revised 15 September 2021; Accepted 16 September 2021; Published 5 October 2021

Academic Editor: Gaetano Luciano

Copyright ( 2021 Mubashir Qayyum and Imbsat Oscar. This is an open access article distributed under the Creative Commons Attribution License, which permits unrestricted use, distribution, and reproduction in any medium, provided the original work is properly cited.

\begin{abstract}
In this study, a new modification of the homotopy perturbation method (HPM) is introduced for various order boundary value problems (BVPs). In this modification, HPM is hybrid with least square optimizer and named as the least square homotopy perturbation method (LSHPM). The proposed scheme is tested against various linear and nonlinear BVPs (second to seventh order DEs). Validity of the obtained solutions is confirmed by finding absolute errors. To analyze the efficiency of the proposed scheme, tested problems have also been solved through HPM and results are compared with LSHPM. Furthermore, obtained results are also compared with other numerical schemes available in literature. Analysis reveals that LSHPM is a consistent and effective scheme which can be used for more complex BVPs in science and engineering.
\end{abstract}

\section{Introduction}

Most of the phenomena in mathematical physics, biological mathematics, and applied mathematics are modeled in the form of differential equations. Solutions to such differential equations are needed to analyze and predict changes in a physical system. In most cases, it is impossible to calculate an exact solution. Therefore, to solve these problems, various numerical and seminumerical methods have been modeled.

Liao presented one of the first analytical methods for nonlinear problems which do not require a small parameter [1]. This method has been applied to many situations in various fields of science and technology [2-4]. In late 90s, Prof. He introduced the homotopy perturbation method (HPM) for highly nonlinear equations [5]. This technique matures into a full fledged theory for nonlinear problems with the efforts of many researchers, notably Ji-Huan $\mathrm{He}$ and his students. This method combines homotopy concepts with perturbation theory and does not rely on small or large parameter like other traditional perturbation techniques [6]. Wide range of problems has been solved using HPM [7-10].
Various modifications of HPM have also been proposed by different researchers to tackle more complex problems. Darvishi et al. combine HPM with adomian polynomials to sine-Gorden type equations in [11]. Biazar and Eslami proposed a modification of HPM based on Taylor series expansion of the kernel and source term [12]. HPM with rank upgrading technique for the superior nonlinear oscillation is proposed in [13]. Qayyum et al. used coupling of HPM with Laplace transform for squeezing flows in [14]. Bota and Caruntu applied HPM with the least square method to fluid flow problems in [15]. For enhanced results, Le-He extension of HPM is proposed in [16]. Ji et al. further applied Le-He extension of HPM to nonlinear packaging system in [17]. Ain et al. introduced Li-He modified extension of HPM for micro-electro-mechanical systems in [18].

In this article, HPM is combined with LS optimizer along with some refine inital guesses to obtain fast convergent semianalytical solutions of linear and nonlinear BVPs. This scheme takes few iterations (cycles) to achieve accurate solution, and hence, it has less computational cost with improved accuracy. 


\section{Basic Idea of LSHPM}

Let us consider the following differential equations along with boundary conditions.

$$
\begin{aligned}
L(\varphi)+N(\varphi)-g(r) & =0, \quad r \in \Omega, \\
B\left(\varphi, \frac{\mathrm{d}^{n} \varphi}{\mathrm{d} r^{n}}\right) & =0, \quad r \in \gamma,
\end{aligned}
$$

where $L$ and $N$ represent the linear and nonlinear parts, $B$ represents the boundary operator, $\gamma$ is the boundary of the domain, $\varphi$ represents an unknown value, and $g(x)$ is a known function. We construct a homotopy such that $\eta(r, p): \varphi \times[0,1] \longrightarrow \mathbb{R}$ which satisfies

$$
\psi(\eta, p)=(1-p)\left[L(\eta)-L\left(\varphi_{0}\right)\right]+p[L(\eta)+N(\eta)-g(r)]=0, \quad r \in \Omega
$$

where $p \in[0,1]$ is the embedding parameter and $L\left(\varphi_{0}\right)$ is the initial guess of equation (1) that satisfies the boundary conditions:

$$
\begin{aligned}
& \psi(\eta, 0)=L(\eta)-L\left(\varphi_{0}\right)=0, \\
& \psi(\eta, 1)=L(\eta)+N(\eta)-g(r)=0 .
\end{aligned}
$$

Therefore, as $p$ changes from 0 to 1 , the solution $\eta(r, p)$ approaches from $\varphi_{0}$ to $\widetilde{\varphi}(r)$. We expand $\eta(r, p)$ into a Taylor series expansion about $p$ to obtain an approximate solution:

$$
\eta(r, p)=\eta_{0}+\sum_{k=1}^{\infty} \eta_{k} p^{k} .
$$

Setting $p=1$, the approximate HPM solution of (1) would be

$$
\widetilde{\varphi}=\lim _{p \longrightarrow 1} \eta(r, p)=\sum_{k=1}^{\infty} \eta_{k} .
$$

We reassign the convergence controlling parameters $c_{i^{\prime}} s$ as coefficients of series (6) and substitute supposed solution $\tilde{\varphi}$ back in (1) to get the residual function:

$$
\mathbb{R}\left(x, c_{i}\right)=R(x, \tilde{\varphi})
$$

Now, we compute the sum of square of residuals:

$$
\mathbb{J}\left(c_{i}\right)=\int_{I} \mathbb{R}^{2}\left(x, c_{i}\right) \mathrm{d} x .
$$

After computing $J\left(c_{i}\right)$, we find the optimal values of $c_{i}^{\prime} s$ from system of equations obtained from $\left(\partial J / \partial c_{i}\right)=0$. Then, putting these optimal values back into $\tilde{\varphi}$ will provide refined solution.

\section{Application of LSHPM}

Problem 1. Second-order linear ODE:

$$
G^{\prime \prime}(x)+2 G^{\prime}(x)+G(x)=0, \quad 0<x<1,
$$

subject to boundary conditions,

$$
\begin{aligned}
& G(0)=1, \\
& G(1)=3 .
\end{aligned}
$$

The exact solution of this problem is $e^{-x}+(3 e-1) x e^{-x}$. First, we construct a homotopy as

$$
(1-p)\left(G^{\prime \prime}(x)\right)+p\left(G^{\prime \prime}(x)+2 G^{\prime}(x)+G(x)\right)=0 .
$$

Zeroth-order problem is

$$
\begin{aligned}
& G_{0}^{\prime \prime}(x)=0, \\
& G_{0}(0)=1, \\
& G_{0}(1)=3 .
\end{aligned}
$$

The solution to (12) is

$$
G_{0}(x)=1+2 x .
$$

First-order problem is

$$
\begin{aligned}
G_{0}(x)+2 G_{0}^{\prime}(x)+G_{1}^{\prime \prime}(x) & =0, \\
G_{1}(0) & =0, \\
G_{1}(1) & =0 .
\end{aligned}
$$

The solution to (14) is

$$
G_{1}(x)=\frac{1}{6}\left(17 x-15 x^{2}-2 x^{3}\right)
$$

Second-order problem is

$$
\begin{aligned}
G_{1}(x)+2 G_{1}^{\prime}(x)+G_{2}^{\prime \prime}(x) & =0, \\
G_{2}(0) & =0, \\
G_{2}(1) & =0 .
\end{aligned}
$$

The solution to (16) is

$$
G_{2}(x)=\frac{1}{360}\left(449 x-1020 x^{2}+430 x^{3}+135 x^{4}+6 x^{5}\right) .
$$

Third-order problem is

$$
\begin{aligned}
G_{2}(x)+2 G_{2}^{\prime}(x)+G_{3}^{\prime \prime}(x) & =0, \\
G_{3}(0) & =0, \\
G_{3}(1) & =0 .
\end{aligned}
$$

The solution to (18) is 


$$
G_{3}(x)=\frac{1}{15120}\left(2351 x-18858 x^{2}+25417 x^{3}-5460 x^{4}-3171 x^{5}-273 x^{6}-6 x^{7}\right)
$$

By combining (13), (15), (17), and (19) will give approximate solution of (9) as follows:

$$
\begin{aligned}
\widetilde{G}(x)= & 1+2 x+\frac{1}{6}\left(17 x-15 x^{2}-2 x^{3}\right)+\frac{1}{360}\left(449 x-1020 x^{2}+430 x^{3}+135 x^{4}+6 x^{5}\right) \\
& +\frac{1}{15120}\left(2351 x-18858 x^{2}+25417 x^{3}-5460 x^{4}-3171 x^{5}-273 x^{6}-6 x^{7}\right) .
\end{aligned}
$$

It follows that (20) consist of $x^{0}, x, x^{2}, x^{4}, x^{5}, x^{6}, x^{7}$, and hence, the required solution is

$$
\widetilde{G}=c_{0}+c_{1} x+c_{2} x^{2}+c_{3} x^{4}+c_{4} x^{5}+c_{5} x^{6}+c_{6} x^{7}
$$

$$
\begin{aligned}
& c_{0}=1, \\
& c_{1}=2-c 2-c 3-c 4-c 5-c 6-c 7 .
\end{aligned}
$$

By applying the boundary conditions given in (27), we have

$$
\widetilde{G}(x)=1+(2-c 2-c 3-c 4-c 5-c 6-c 7) x+c_{2} x^{2}+c_{3} x^{4}+c_{4} x^{5}+c_{5} x^{6}+c_{6} x^{7}
$$

Now, replacing $G(x)$ with $\widetilde{G}(x)$ in $(27)$

$$
\begin{aligned}
R\left(x, c_{2}, c_{3}, c_{4}, c_{5}, c_{6}, c_{7}\right)= & 1+2 c_{2}+6 c_{3} x+\left(2-c_{2}-c_{3}-c_{4}-c_{5}-c_{6}-c_{7}\right) x+c_{2} x^{2}+12 c_{4} x^{2} \\
& +c_{3} x^{3}+20 c_{5} x^{3}+c_{4} x^{4}+30 c_{6} x^{4}+c_{5} x^{5}+42 c_{7} x^{5}+c_{6} x^{6}+c_{7} x^{7} \\
& +2\left(2-c_{2}-c_{3}-c_{4}-c_{5}-c_{6}-c_{7}+2 c_{2} x+3 c_{3} x^{2}+4 c_{4} x^{3}+5 c_{5} x^{4}+6 c_{6} x^{5}+7 c_{7} x^{6}\right) . \\
& G^{\prime \prime}(x)+G(x)^{2}-x^{4}-2=0, \quad 0<x<1,
\end{aligned}
$$

Next, we minimize $J\left(c_{2}, c_{3}, c_{4}, c_{5}, c_{6}\right)=\int_{0}^{1} R^{2}\left(c_{2}, c_{3}\right.$, $\left.c_{4}, c_{5}, c_{6}\right) \mathrm{d} x$ to get optimal values of $c_{i}^{\prime} s$. Here,

$$
\begin{aligned}
& c_{2}=-6.65482, \\
& c_{3}=3.4104, \\
& c_{4}=-1.14897, \\
& c_{5}=0.285225, \\
& c_{6}=-0.0522252, \\
& c_{7}=0.00554595 .
\end{aligned}
$$

The third-order approximation solution is

$$
\begin{aligned}
\widetilde{G}(x)= & 1+6.15485 x-6.65482 x^{2}+3.4104 x^{3}-1.14897 x^{4} \\
& +0.285225 x^{5}-0.0522252 x^{6}+0.00554595 x^{7}
\end{aligned}
$$

The results are presented in Table 1 .

Problem 2. Let us consider the following second-order nonhomogeneous nonlinear ODE [20]: subject to boundary conditions,

$$
\begin{aligned}
& G(0)=0, \\
& G(1)=1 .
\end{aligned}
$$

This problem was studied by Momani [20] by applying the differential transform method, and later, Mohamad [19] by applying four different numerical methods for this problem. The exact solution to this problem is $x^{2}$.

The first step is to construct a homotopy:

$$
(1-p)\left(G^{\prime \prime}(x)\right)+p\left(G^{\prime \prime}(x)+G(x)^{2}+x^{4}-2\right)=0 \text {. }
$$

Then, we linearize the problem as follows.

Zeroth-order problem is

$$
\begin{aligned}
& G_{0}^{\prime \prime}(x)=0, \\
& G_{0}(0)=0, \\
& G_{0}(1)=1 .
\end{aligned}
$$

The solution to (30) is 
TABLe 1: Comparison of third-order absolute error of LSHPM with HPM in Problem 1.

\begin{tabular}{lccccc}
\hline$x$ & $\begin{array}{c}\text { Exact } \\
\text { Solution }\end{array}$ & Solution & LSHPM & \multicolumn{2}{c}{ HPM } \\
\hline 0 & 1 & 1. & Error & Solution & 1 \\
0.1 & 1.55223 & 1.55223 & $3.76 \times 10^{-8}$ & 1.56034 & 0 \\
0.2 & 1.99031 & 1.99031 & $4.82 \times 10^{-8}$ & 2.00428 & $2.10 \times 10^{-3}$ \\
0.3 & 2.33095 & 2.33095 & $6.86 \times 10^{-8}$ & 2.60253 & $1.39 \times 10^{-2}$ \\
0.4 & 2.58873 & 2.58873 & $3.12 \times 10^{-8}$ & 2.7852 & $1.37 \times 10^{-2}$ \\
0.5 & 2.77635 & 2.77635 & $9.38 \times 10^{-8}$ & 2.90766 & $8.85 \times 10^{-3}$ \\
0.6 & 2.90481 & 2.90481 & $2.57 \times 10^{-8}$ & 2.98144 & $2.85 \times 10^{-3}$ \\
0.7 & 2.98368 & 2.98368 & $6.94 \times 10^{-8}$ & 3.01646 & $2.23 \times 10^{-3}$ \\
0.8 & 3.02123 & 3.02123 & $4.41 \times 10^{-8}$ & 3.02073 & $3.76 \times 10^{-3}$ \\
0.9 & 3.02462 & 3.02462 & $3.67 \times 10^{-8}$ & 0 & $3.88 \times 10^{-3}$ \\
1 & 3 & 3 & 0 & 0 \\
\hline
\end{tabular}

$$
G_{0}(x)=x .
$$

First-order problem is

$$
\begin{aligned}
-2+x^{4}+G_{0}(x)^{2}+G_{1}^{\prime \prime}(x) & =0, \\
G_{1}(0) & =0, \\
G_{1}(1) & =0 .
\end{aligned}
$$

The solution to (32) is

$$
G_{1}(x)=\frac{1}{60}\left(-53 x+60 x^{2}-5 x^{4}-2 x^{6}\right)
$$

Second-order problem is

$$
\begin{aligned}
2 G_{0}(x) G_{1}(x)+G_{2}^{\prime \prime}(x) & =0, \\
G_{2}(0) & =0, \\
G_{2}(1) & =0 .
\end{aligned}
$$

The solution to (34) is

$G_{2}(x)=\frac{1}{7560}\left(-394 x+1113 x^{4}-756 x^{5}+30 x^{7}+7 x^{9}\right)$.

By combining (31), (33), and (35) will give an approximate series solution of (27) as

$$
\widetilde{G}(x)=x+\frac{1}{60}\left(-53 x+60 x^{2}-5 x^{4}-2 x^{6}\right)+\frac{1}{7560}\left(-394 x+1113 x^{4}-756 x^{5}+30 x^{7}+7 x^{9}\right) .
$$

(36) consists of $x, x^{2}, x^{4}, x^{5}, x^{6}, x^{7}, x^{9}$; hence, the required solution will take the form

$$
\widetilde{G}(x)=c_{1} x+c_{2} x^{2}+c_{3} x^{4}+c_{4} x^{5}+c_{5} x^{6}+c_{6} x^{7}+c_{7} x^{9} .
$$

By applying boundary conditions from (27) gives

$$
c_{1}=1-1\left(c_{2}+c_{3}+c_{4}+c_{5}+c_{6}+c_{7}\right) \text {. }
$$

$$
\widetilde{G}(x)=\left(1-1\left(c_{2}+c_{3}+c_{4}+c_{5}+c_{6}+c_{7}\right)\right) x+c_{2} x^{2}+c_{3} x^{4}+c_{4} x^{5}+c_{5} x^{6}+c_{6} x^{7}+c_{7} x^{9} .
$$

Now, replacing $G(x)$ with $\widetilde{G}(x)$ in (27), we get the following residual function:

$$
\begin{aligned}
R\left(x, c_{2}, c_{3}, c_{4}, c_{5}, c_{6}, c_{7}\right)= & -2+2 c_{2}+12 c_{3} x^{2}+20 c_{4} x^{3}+x^{4}+30 c_{5} x^{4}+42 c_{6} x^{5} \\
& +72 c_{7} x^{7}+\left(\left(1-c_{2}-c_{3}-c_{4}-c_{5}-c_{6}-c_{7}\right) x+c_{2} x^{2}+c_{3} x^{4}+c_{4} x^{5}+c_{5} x^{6}+c_{6} x^{7}+c_{7} x^{9}\right)^{2} .
\end{aligned}
$$

Next, we minimize $J\left(c_{2}, c_{3}, c_{4}, c_{5}, c_{6}, c_{7}\right)=\int_{0}^{1} R^{2}\left(c_{2}, c_{3}\right.$, $\left.c_{4}, c_{5}, c_{6}, c_{7}\right) \mathrm{d} x$ to get optimal values of $c_{i}^{\prime} s$. 
Here,

Hence, second-order approximate solution is

$$
\begin{aligned}
& c_{1}=0.0725613, \\
& c_{2}=0.999994, \\
& c_{3}=-0.0000429997, \\
& c_{4}=-0.00942955, \\
& c_{5}=-0.0618513, \\
& c_{6}=-0.00444548, \\
& c_{7}=0.00321356 .
\end{aligned}
$$

$$
\begin{aligned}
\widetilde{G}(x)= & 0.0725613 x+0.999994 x^{2}-0.0000429997 x^{4}-0.00942955 x^{5} \\
& -0.0618513 x^{6}-0.00444548 x^{7}+0.00321356 x^{9} .
\end{aligned}
$$

The results are presented in Table 2.

$$
\begin{aligned}
G(0) & =0, \\
G^{\prime}(0) & =1, \\
G(1) & =0 .
\end{aligned}
$$

Problem 3. Third-order linear ODE [21]:

$$
G^{\prime \prime \prime}(x)-x G(x)-\left(x^{3}-2 x^{2}-5 x-3\right) e^{x}, \quad 0<x<1,
$$

subject to boundary conditions

The exact solution to this problem is $x(1-x) e^{x}$. After applying LSHPM proceedure, second-order approximate solution is

$$
\begin{aligned}
\widetilde{G}(x)= & -26.4063+26.4063 e^{x}-14.2359 x-11.1703 e^{x} x-2.77751 x^{2} \\
& +0.744709 e^{x} x^{2}-0.0605874 e^{x} x^{3}+0.118979 x^{4}-0.00260775 e^{x} x^{4} \\
& +0.029123 x^{5}+0.00352613 x^{6}-0.0000251847 x^{9}-3.92104 \times 10^{-7} x^{10} .
\end{aligned}
$$

The results are presented in Table 3 .

$$
\begin{aligned}
G(1) & =0, \\
G^{\prime}(1) & =-2, \\
G^{\prime}(0) & =0 .
\end{aligned}
$$

Problem 4. Third-order nonlinear ODE [24]:

$$
G^{\prime \prime \prime}(x)-G^{2}(x)+G(x)+x^{2}\left(x^{2}-1\right), \quad 0<x<1,
$$

The exact solution to this problem is $1-x^{2}$. The firstsubject to boundary conditions order approximate solution using LSHPM is

$$
\begin{aligned}
\widetilde{G}(x)= & 1-x^{2}+1.45333 \times 10^{-18} x^{3}-2.76238 \times 10^{-17} x^{5}+3.64818 \times 10^{-16} x^{7} \\
& -7.51562 \times 10^{-16} x^{8}+1.13156 \times 10^{-15} x^{10}-2.20449 \times 10^{-15} x^{12} \\
& +1.83775 \times 10^{-15} x^{13}-4.11003 \times 10^{-16} x^{15}+5.91342 \times 10^{-17} x^{17} .
\end{aligned}
$$

The results are presented in Table 4.

Problem 5. Fourth-order linear ODE [25]:

$$
G^{(i v)}(x)-G^{\prime \prime}(x)-G(x)-e^{x}(x-3)=0, \quad 0<x<1,
$$

subject to boundary conditions

$$
\begin{aligned}
G(0) & =1, \\
G^{\prime}(0) & =0, \\
G(1) & =0, \\
G^{\prime}(1) & =-e .
\end{aligned}
$$


TABLE 2: Comparison of second-order absolute error of LSHPM with HPM and RK4 in Problem 2.

\begin{tabular}{lcccccr}
\hline$x$ & Exact & \multicolumn{2}{c}{ LSHPM } & & HPM & RK4 \\
Solution & Solution & Error & Solution & 0.00886897 & $1.13 \times 10^{-3}$ & $2.14 \times 10^{-8}$ \\
\hline 0.1 & 0.01 & 0.01 & $4.11 \times 10^{-16}$ & 0.0378151 & $2.18 \times 10^{-3}$ & $8.56 \times 10^{-8}$ \\
0.2 & 0.04 & 0.04 & $1.04 \times 10^{-15}$ & 0.086977 & $3.02 \times 10^{-3}$ & $1.86 \times 10^{-7}$ \\
0.3 & 0.09 & 0.09 & $1.04 \times 10^{-15}$ & 0.156489 & $3.51 \times 10^{-3}$ & $3.42 \times 10^{-7}$ \\
0.4 & 0.16 & 0.16 & $5.55 \times 10^{-16}$ & 0.246425 & $3.57 \times 10^{-3}$ & $5.06 \times 10^{-7}$ \\
0.5 & 0.25 & 0.25 & $1.38 \times 10^{-16}$ & 0.356776 & $3.22 \times 10^{-3}$ & $7.45 \times 10^{-7}$ \\
0.6 & 0.36 & 0.36 & $1.66 \times 10^{-16}$ & 0.487449 & $2.55 \times 10^{-3}$ & $1.07 \times 10^{-6}$ \\
0.7 & 0.49 & 0.49 & $3.88 \times 10^{-16}$ & 0.638298 & $1.70 \times 10^{-3}$ & $1.43 \times 10^{-6}$ \\
0.8 & 0.64 & 0.64 & $3.33 \times 10^{-16}$ & 0.809174 & $8.25 \times 10^{-4}$ & $1.90 \times 10^{-6}$ \\
0.9 & 0.81 & 0.81 & 0. & 1. & 0. & $2.38 \times 10^{-6}$ \\
1. & 1. & 1. & 0. & & & \\
\hline
\end{tabular}

TABLE 3: Comparison of second-order absolute error of LSHPM with HPM, Q-spline, and B-spline in Problem 3.

\begin{tabular}{|c|c|c|c|c|c|c|c|}
\hline \multirow{2}{*}{$x$} & \multirow{2}{*}{$\begin{array}{c}\text { Exact } \\
\text { Solution }\end{array}$} & \multicolumn{2}{|c|}{ LSHPM } & \multicolumn{2}{|c|}{ HPM } & \multirow{2}{*}{$\begin{array}{l}\text { Q-spline } \\
\text { Error [22] }\end{array}$} & \multirow{2}{*}{$\begin{array}{c}\text { B-spline } \\
\text { Error [23] }\end{array}$} \\
\hline & & Solution & Error & Solution & Error & & \\
\hline 0 & 0 & $-1.77 \times 10^{-15}$ & $1.77 \times 10^{-15}$ & 0 & 0 & 0 & 0 \\
\hline 0.1 & 0.0994654 & 0.0994654 & $3.36 \times 10^{-10}$ & 0.0994651 & $3.05 \times 10^{-7}$ & $1 \times 10^{-7}$ & 0 \\
\hline 0.2 & 0.195424 & 0.195424 & $5.43 \times 10^{-10}$ & 0.195423 & $1.22 \times 10^{-6}$ & $2 \times 10^{-7}$ & $2 \times 10^{-3}$ \\
\hline 0.3 & 0.28347 & 0.28347 & $2.01 \times 10^{-10}$ & 0.283468 & $2.72 \times 10^{-6}$ & $3 \times 10^{-7}$ & $5 \times 10^{-3}$ \\
\hline 0.4 & 0.358038 & 0.358038 & $7.95 \times 10^{-10}$ & 0.358033 & $4.73 \times 10^{-6}$ & $5 \times 10^{-7}$ & $7 \times 10^{-3}$ \\
\hline 0.5 & 0.41218 & 0.41218 & $4.16 \times 10^{-10}$ & 0.412173 & $7.04 \times 10^{-6}$ & $6 \times 10^{-7}$ & $8 \times 10^{-3}$ \\
\hline 0.6 & 0.437309 & 0.437309 & $3.07 \times 10^{-10}$ & 0.437299 & $9.25 \times 10^{-6}$ & $7 \times 10^{-7}$ & $8 \times 10^{-3}$ \\
\hline 0.7 & 0.422888 & 0.422888 & $4.05 \times 10^{-10}$ & 0.422877 & $1.06 \times 10^{-5}$ & $7 \times 10^{-7}$ & $7 \times 10^{-3}$ \\
\hline 0.8 & 0.356087 & 0.356087 & $1.68 \times 10^{-11}$ & 0.356076 & $1.03 \times 10^{-5}$ & $7 \times 10^{-7}$ & $6 \times 10^{-3}$ \\
\hline 0.9 & 0.221364 & 0.221364 & $1.17 \times 10^{-10}$ & 0.221357 & $7.15 \times 10^{-6}$ & $4 \times 10^{-7}$ & $4 \times 10^{-3}$ \\
\hline 1 & 0 & $-7.52 \times 10^{-15}$ & $7.52 \times 10^{-15}$ & $-1.59 \times 10^{-13}$ & $1.59 \times 10^{-13}$ & 0 & $1.4 \times 10^{-2}$ \\
\hline
\end{tabular}

TABLE 4: Comparison of second-order absolute error of LSHPM with HPM and MADM in Problem 4.

\begin{tabular}{|c|c|c|c|c|c|c|}
\hline \multirow{2}{*}{$x$} & \multirow{2}{*}{$\begin{array}{c}\text { Exact } \\
\text { Solution }\end{array}$} & \multicolumn{2}{|c|}{ LSHPM } & \multicolumn{2}{|c|}{ HPM } & \multirow{2}{*}{$\begin{array}{c}\text { MADM [24] } \\
\text { Error }\end{array}$} \\
\hline & & Solution & Error & Solution & Error & \\
\hline 0.1 & 0.99 & 0.99 & 0 & 0.990368 & $3.67 \times 10^{-4}$ & $2.78 \times 10^{-3}$ \\
\hline 0.2 & 0.96 & 0.96 & 0 & 0.960324 & $3.23 \times 10^{-4}$ & $3.44 \times 10^{-3}$ \\
\hline 0.3 & 0.91 & 0.91 & 0 & 0.910265 & $2.65 \times 10^{-4}$ & $6.41 \times 10^{-3}$ \\
\hline 0.4 & 0.84 & 0.84 & 0 & 0.840202 & $2.02 \times 10^{-4}$ & $7.05 \times 10^{-3}$ \\
\hline 0.5 & 0.75 & 0.75 & 0 & 0.750142 & $1.42 \times 10^{-4}$ & $6.14 \times 10^{-3}$ \\
\hline 0.6 & 0.64 & 0.64 & 0 & 0.640091 & $9.11 \times 10^{-5}$ & $4.37 \times 10^{-3}$ \\
\hline 0.7 & 0.51 & 0.51 & 0 & 0.510051 & $5.08 \times 10^{-5}$ & $2.31 \times 10^{-3}$ \\
\hline 0.8 & 0.36 & 0.36 & $1.11 \times 10^{-16}$ & 0.360022 & $22 \times 10^{-5}$ & $3.98 \times 10^{-4}$ \\
\hline 0.9 & 0.19 & 0.19 & 0 & 0.190006 & $5.57 \times 10^{-6}$ & $1.04 \times 10^{-3}$ \\
\hline 1 & 0 & 0 & 0 & 0 & 0 & $1.79 \times 10^{-3}$ \\
\hline
\end{tabular}

TABLE 5: Comparison of first-order absolute error of LSHPM with HPM and VIM in Problem 5.

\begin{tabular}{|c|c|c|c|c|c|c|}
\hline \multirow{2}{*}{$x$} & \multirow{2}{*}{$\begin{array}{c}\text { Exact } \\
\text { Solution }\end{array}$} & \multicolumn{2}{|c|}{ LSHPM } & \multicolumn{2}{|c|}{ HPM } & \multirow{2}{*}{$\begin{array}{c}\text { VIM [25] } \\
\text { Error }\end{array}$} \\
\hline & & Solution & Error & Solution & Error & \\
\hline 0 & 1 & 1 & 0 & 1 & 0 & 0 \\
\hline 0.1 & 0.994654 & 0.994654 & 0 & 0.99462 & $3.37 \times 10^{-5}$ & $2.00 \times 10^{-10}$ \\
\hline 0.2 & 0.977122 & 0.977122 & 0 & 0.977 & $1.21 \times 10^{-4}$ & $7.00 \times 10^{-10}$ \\
\hline 0.3 & 0.944901 & 0.944901 & 0 & 0.944672 & $2.29 \times 10^{-4}$ & $1.35 \times 10^{-9}$ \\
\hline 0.4 & 0.895095 & 0.895095 & 0 & 0.894777 & $3.17 \times 10^{-4}$ & $2.00 \times 10^{-9}$ \\
\hline 0.5 & 0.824361 & 0.824361 & 0 & 0.824006 & $3.54 \times 10^{-4}$ & $2.50 \times 10^{-9}$ \\
\hline 0.6 & 0.728848 & 0.728848 & 0 & 0.728521 & $3.26 \times 10^{-4}$ & $2.72 \times 10^{-9}$ \\
\hline 0.7 & 0.604126 & 0.604126 & 0 & 0.603883 & $2.42 \times 10^{-4}$ & $2.21 \times 10^{-9}$ \\
\hline 0.8 & 0.445108 & 0.445108 & 0 & 0.444976 & $1.32 \times 10^{-4}$ & $1.80 \times 10^{-9}$ \\
\hline 0.9 & 0.24596 & 0.24596 & 0 & 0.245923 & $3.74 \times 10^{-5}$ & $7.25 \times 10^{-10}$ \\
\hline 1 & 0 & 0 & 0 & $5.55112 \times 10^{-16}$ & $5.55 \times 10^{-16}$ & $9.94 \times 10^{-14}$ \\
\hline
\end{tabular}


TABLE 6: Comparison of second-order absolute error of LSHPM with HPM in Problem 6.

\begin{tabular}{lcccc}
\hline$x$ & & LSHPM & & HPM \\
& Solution & Error & Solution & 0 \\
0 & 0 & 0 & 0.150157 & $0.54 \times 10^{-5}$ \\
0.1 & 0.150158 & $5.14 \times 10^{-7}$ & 0.297237 & $5.10 \times 10^{-5}$ \\
0.2 & 0.297237 & $5.99 \times 10^{-7}$ & 0.438169 & $7.55 \times 10^{-5}$ \\
0.3 & 0.43817 & $1.56 \times 10^{-7}$ & 0.569899 & $9.58 \times 10^{-5}$ \\
0.4 & 0.5699 & $4.59 \times 10^{-7}$ & 0.689396 & $1.06 \times 10^{-4}$ \\
0.5 & 0.689397 & $6.55 \times 10^{-7}$ & 0.793661 & $9.88 \times 10^{-5}$ \\
0.6 & 0.793661 & $1.16 \times 10^{-7}$ & 0.879734 & $6.54 \times 10^{-5}$ \\
0.7 & 0.879734 & $6.64 \times 10^{-7}$ & 0.944705 & $2.03 \times 10^{-6}$ \\
0.8 & 0.944705 & $4.64 \times 10^{-7}$ & 0.985722 & $8.25 \times 10^{-5}$ \\
0.9 & 0.985722 & $9.06 \times 10^{-7}$ & 1 & $1.51 \times 10^{-4}$ \\
1 & 1 & $2.46 \times 10^{-6}$ & & \\
\hline
\end{tabular}

TABLE 7: Comparison of second-order absolute error of LSHPM with HPM, OHAM, and B-spline in Problem 7.

\begin{tabular}{|c|c|c|c|c|c|c|c|}
\hline \multirow{2}{*}{$x$} & \multirow{2}{*}{$\begin{array}{c}\text { Exact } \\
\text { Solution }\end{array}$} & \multicolumn{2}{|c|}{ LSHPM } & \multicolumn{2}{|c|}{ HPM } & \multirow{2}{*}{$\begin{array}{c}\text { OHAM } \\
\text { Error [27] }\end{array}$} & \multirow{2}{*}{$\begin{array}{l}\text { B-spline } \\
\text { Error [28] }\end{array}$} \\
\hline & & Solution & Error & Solution & Error & & \\
\hline 0 & 0 & 0 & 0 & 0 & 0 & 0 & 0 \\
\hline 0.1 & 0.0994654 & 0.0994654 & $1.57 \times 10^{-14}$ & 0.0994654 & $4.85 \times 10^{-12}$ & $9 \times 10^{-11}$ & $8 \times 10^{-3}$ \\
\hline 0.2 & 0.195424 & 0.195424 & $3.63 \times 10^{-13}$ & 0.195424 & $3.26 \times 10^{-11}$ & $4 \times 10^{-10}$ & $1 \times 10^{-3}$ \\
\hline 0.3 & 0.28347 & 0.28347 & $1.24 \times 10^{-12}$ & 0.28347 & $8.99 \times 10^{-11}$ & $5 \times 10^{-10}$ & $5 \times 10^{-3}$ \\
\hline 0.4 & 0.358038 & 0.358038 & $1.75 \times 10^{-12}$ & 0.358038 & $1.66 \times 10^{-10}$ & $2 \times 10^{-11}$ & $3 \times 10^{-3}$ \\
\hline 0.5 & 0.41218 & 0.41218 & $8.38 \times 10^{-13}$ & 0.41218 & $2.38 \times 10^{-10}$ & $1 \times 10^{-9}$ & $8 \times 10^{-3}$ \\
\hline 0.6 & 0.437309 & 0.437309 & $7.74 \times 10^{-13}$ & 0.437309 & $2.76 \times 10^{-10}$ & $2 \times 10^{-9}$ & $6 \times 10^{-3}$ \\
\hline 0.7 & 0.422888 & 0.422888 & $1.35 \times 10^{-12}$ & 0.422888 & $2.56 \times 10^{-10}$ & $2 \times 10^{-9}$ & 0 \\
\hline 0.8 & 0.356087 & 0.356087 & $6.56 \times 10^{-13}$ & 0.356087 & $1.74 \times 10^{-10}$ & $1 \times 10^{-9}$ & $9 \times 10^{-3}$ \\
\hline 0.9 & 0.221364 & 0.221364 & $7.23 \times 10^{-14}$ & 0.221364 & $6.34 \times 10^{-11}$ & $4 \times 10^{-10}$ & $9 \times 10^{-3}$ \\
\hline 1 & 0 & $8.56 \times 10^{-15}$ & $8.56 \times 10^{-15}$ & $-6.57 \times 10^{-14}$ & $6.57 \times 10^{-14}$ & 0 & 0 \\
\hline
\end{tabular}

The exact solution to this problem is $e^{x}-x e^{x}$. The firstorder approximate solution using LSHPM is

$$
\widetilde{G}(x)=e^{x}-x e^{x} \text {. }
$$

The results are presented in Table 5 .

Problem 6. Fourth-order nonlinear ODE [26]:

$$
G^{(i v)}(x)+R\left[(x-G(x)) G^{\prime \prime \prime}(x)+3 G^{\prime \prime}(x)\right]=0, \quad 0<x<1,
$$

subject to boundary conditions

$$
\begin{aligned}
G(1) & =1, \\
G^{\prime}(1) & =0, \\
G(0) & =0, \\
G^{\prime \prime}(0) & =0,
\end{aligned}
$$

where $R$ is the Reynolds number, and we have fixed it to $R=0.1$. The second-order approximate solution of this problem using LSHPM is

$$
\begin{aligned}
\widetilde{G}(x)= & 1.50671 x-0.513264 x^{3}+0.00639854 x^{5}+0.00016573 x^{7} \\
& -7.3875 \times 10^{-6} x^{9}-1.43995 \times 10^{-7} x^{11} .
\end{aligned}
$$

TABLE 8: Comparison of zeroth-order absolute error of LSHPM with HPM in Problem 8.

\begin{tabular}{lccccc}
\hline \multirow{2}{*}{$x$} & Exact & \multicolumn{2}{c}{ LSHPM } & \multicolumn{2}{c}{ HPM } \\
& Solution & Solution & Error & Solution & Error \\
\hline 0 & 1.60944 & 1.60944 & 0 & 1.60944 & 0 \\
0.1 & 1.62924 & 1.62924 & $1.50 \times 10^{-9}$ & 1.62924 & $2.36 \times 10^{-6}$ \\
0.2 & 1.64866 & 1.64866 & $9.38 \times 10^{-9}$ & 1.64867 & $1.56 \times 10^{-5}$ \\
0.3 & 1.66771 & 1.66771 & $2.38 \times 10^{-8}$ & 1.66775 & $4.22 \times 10^{-5}$ \\
0.4 & 1.6864 & 1.6864 & $4.09 \times 10^{-8}$ & 1.68648 & $7.67 \times 10^{-5}$ \\
0.5 & 1.70475 & 1.70475 & $5.46 \times 10^{-8}$ & 1.70486 & $1.08 \times 10^{-4}$ \\
0.6 & 1.72277 & 1.72277 & $5.95 \times 10^{-8}$ & 1.72289 & $1.24 \times 10^{-4}$ \\
0.7 & 1.74047 & 1.74047 & $5.24 \times 10^{-8}$ & 1.74058 & $1.15 \times 10^{-4}$ \\
0.8 & 1.75786 & 1.75786 & $3.42 \times 10^{-8}$ & 1.75794 & $7.96 \times 10^{-5}$ \\
0.9 & 1.77495 & 1.77495 & $1.20 \times 10^{-8}$ & 1.77498 & $2.93 \times 10^{-5}$ \\
1 & 1.79176 & 1.79176 & 0 & 1.79176 & 0 \\
\hline
\end{tabular}

The results are presented in Table 6.

Problem 7. Fifth-order linear ODE [26]:

$$
G^{(v)}(x)-G(x)+15 e^{x}+10 x e^{x}=0, \quad 0<x<1,
$$

subject to boundary conditions 
TABLE 9: Comparison of first-order absolute error of LSHPM with second-order HPM, HPLM, and ADM in Problem 9.

\begin{tabular}{|c|c|c|c|c|c|c|c|}
\hline \multirow{2}{*}{$x$} & \multirow{2}{*}{$\begin{array}{c}\text { Exact } \\
\text { Solution }\end{array}$} & \multicolumn{2}{|c|}{ LSHPM } & \multicolumn{2}{|c|}{ HPM } & \multirow{2}{*}{$\begin{array}{c}\text { HPLM } \\
\text { Error [14] }\end{array}$} & \multirow{2}{*}{$\begin{array}{c}\text { ADM } \\
\text { Error [30] }\end{array}$} \\
\hline & & Solution & Error & Solution & Error & & \\
\hline 0 & 1 & 1 & 0 & 1 & 0 & 0 & 0 \\
\hline 0.1 & 0.994654 & 0.994654 & $1.88 \times 10^{-15}$ & 0.994658 & $3.98 \times 10^{-6}$ & $1.1 \times 10^{-13}$ & $4.0 \times 10^{-4}$ \\
\hline 0.2 & 0.977122 & 0.977122 & $7.77 \times 10^{-16}$ & 0.97713 & $7.58 \times 10^{-6}$ & $2.2 \times 10^{-13}$ & $7.7 \times 10^{-4}$ \\
\hline 0.3 & 0.944901 & 0.944901 & $1.99 \times 10^{-15}$ & 0.944912 & $1.04 \times 10^{-5}$ & $3.0 \times 10^{-13}$ & $1.0 \times 10^{-3}$ \\
\hline 0.4 & 0.895095 & 0.895095 & $8.21 \times 10^{-15}$ & 0.895107 & $1.22 \times 10^{-5}$ & $3.6 \times 10^{-13}$ & $1.2 \times 10^{-3}$ \\
\hline 0.5 & 0.824361 & 0.824361 & $1.33 \times 10^{-14}$ & 0.824374 & $1.28 \times 10^{-5}$ & $3.9 \times 10^{-13}$ & $1.3 \times 10^{-3}$ \\
\hline 0.6 & 0.728848 & 0.728848 & $1.60 \times 10^{-14}$ & 0.72886 & $1.22 \times 10^{-5}$ & $3.7 \times 10^{-13}$ & $1.2 \times 10^{-3}$ \\
\hline 0.7 & 0.604126 & 0.604126 & $8.99 \times 10^{-15}$ & 0.604136 & $1.04 \times 10^{-5}$ & $3.2 \times 10^{-13}$ & $1.0 \times 10^{-3}$ \\
\hline 0.8 & 0.445108 & 0.445108 & $1.11 \times 10^{-15}$ & 0.445116 & $7.58 \times 10^{-6}$ & $2.4 \times 10^{-13}$ & $4.0 \times 10^{-4}$ \\
\hline 0.9 & 0.24596 & 0.24596 & $2.41 \times 10^{-15}$ & 0.245964 & $3.98 \times 10^{-6}$ & $1.3 \times 10^{-13}$ & $7.7 \times 10^{-4}$ \\
\hline 1 & 0 & $-3.19 \times 10^{-16}$ & $3.19 \times 10^{-16}$ & $1.59 \times 10^{-16}$ & $1.59 \times 10^{-16}$ & $1.9 \times 10^{-15}$ & 0 \\
\hline
\end{tabular}

$$
\begin{aligned}
G(0) & =0, \\
G^{\prime}(0) & =1, \\
G^{\prime \prime}(0) & =0, \\
G(1) & =0, \\
G^{\prime}(1) & =-e .
\end{aligned}
$$

The exact solution of this problem is $\left(x-x^{2}\right) e^{x}$. The second-order approximate solution using LSHPM is
The results are presented in Table 7.

$$
\begin{aligned}
\widetilde{G}(x)= & -36.8878+36.8878 e^{x}-24.1329 x-11.7548 e^{x} x-6.68904 x^{2}-0.770542 x^{3} \\
& +0.0888158 x^{4}+0.0573868 x^{5}+0.0133906 x^{6}+0.00206275 x^{7}+0.000226806 x^{8} \\
& +0.0000165216 x^{9}-1.3145 \times 10^{-7} x^{11}-2.34824 \times 10^{-8} x^{13}+2.1814 \times 10^{-9} x^{14} .
\end{aligned}
$$

$$
\begin{aligned}
\widetilde{G}(x)= & 0.0644833+0.00801314 x-0.000801314 x^{2}+0.00010495 x^{3} \\
& -0.0000119326 x^{4}-2.38377 \times 10^{-9} x^{6}+0.959934 \ln [5+x]
\end{aligned}
$$

Problem 8. Fifth-order nonlinear ODE [29]:

$$
G^{(v)}(x)-\frac{\left(G^{\prime}(x)\right)^{2}}{(5+x)^{3}}-\frac{23}{(5+x)^{5}}=0, \quad 0<x<1,
$$

along with boundary conditions

$$
\begin{aligned}
G(0) & =\ln (5), \\
G^{\prime}(0) & =\frac{1}{5}, \\
G^{\prime \prime}(0) & =-\frac{1}{25}, \\
G(1) & =\ln (6), \\
G^{\prime}(1) & =\frac{1}{6} .
\end{aligned}
$$

The exact solution to this problem is $\ln (x+5)$. The zeroth-order approximate solution using LSHPM is
The results are presented in Table 8.

Problem 9. Sixth-order linear ODE [14]:

$$
G^{(v i)}(x)-G(x)+6 e^{x}=0, \quad 0<x<1,
$$

subject to boundary conditions

$$
\begin{aligned}
G(0) & =1, \\
G^{\prime \prime}(0) & =-1, \\
G^{i v}(0) & =-3, \\
G(1) & =0, \\
G^{\prime \prime}(1) & =-2 e, \\
G^{i v}(1) & =-4 e .
\end{aligned}
$$

The exact solution to this problem is $G(x)=(1-x) e^{x}$. The first-order approximate solution using LSHPM is 
TABLE 10: Comparison of zeroth-order absolute error of LSHPM with second-order HPM, VIM, and ADM in Problem 10.

\begin{tabular}{|c|c|c|c|c|c|c|c|}
\hline \multirow{2}{*}{$x$} & \multirow{2}{*}{$\begin{array}{c}\text { Exact } \\
\text { Solution }\end{array}$} & \multicolumn{2}{|c|}{ LSHPM } & \multicolumn{2}{|c|}{ HPM } & \multirow{2}{*}{$\begin{array}{c}\text { VIM } \\
\text { Error [31] }\end{array}$} & \multirow{2}{*}{$\begin{array}{c}\text { ADM } \\
\text { Error [30] }\end{array}$} \\
\hline & & Solution & Error & Solution & Error & & \\
\hline 0 & 1 & 1 & 0 & 1 & 0 & 0 & 0 \\
\hline 0.1 & 1.10517 & 1.10516 & $1.22 \times 10^{-5}$ & 1.10561 & $4.43 \times 10^{-4}$ & $1.2 \times 10^{-4}$ & $1.2 \times 10^{-4}$ \\
\hline 0.2 & 1.2214 & 1.22138 & $2.32 \times 10^{-5}$ & 1.22225 & $8.44 \times 10^{-4}$ & $2.3 \times 10^{-4}$ & $2.3 \times 10^{-4}$ \\
\hline 0.3 & 1.34986 & 1.34983 & $3.21 \times 10^{-5}$ & 1.35102 & $1.16 \times 10^{-3}$ & $3.2 \times 10^{-4}$ & $3.2 \times 10^{-4}$ \\
\hline 0.4 & 1.49182 & 1.49179 & $3.78 \times 10^{-5}$ & 1.49319 & $1.36 \times 10^{-3}$ & $3.8 \times 10^{-4}$ & $3.8 \times 10^{-4}$ \\
\hline 0.5 & 1.64872 & 1.64868 & $3.99 \times 10^{-5}$ & 1.65016 & $1.44 \times 10^{-3}$ & $4.0 \times 10^{-4}$ & $4.0 \times 10^{-4}$ \\
\hline 0.6 & 1.82212 & 1.82208 & $3.79 \times 10^{-5}$ & 1.82349 & $1.37 \times 10^{-3}$ & $3.9 \times 10^{-4}$ & $3.9 \times 10^{-4}$ \\
\hline 0.7 & 2.01375 & 2.01372 & $3.22 \times 10^{-5}$ & 2.01492 & $1.16 \times 10^{-3}$ & $3.3 \times 10^{-4}$ & $3.3 \times 10^{-4}$ \\
\hline 0.8 & 2.22554 & 2.22552 & $2.34 \times 10^{-5}$ & 2.22639 & $8.51 \times 10^{-4}$ & $2.4 \times 10^{-4}$ & $2.4 \times 10^{-4}$ \\
\hline 0.9 & 2.4596 & 2.45959 & $1.22 \times 10^{-5}$ & 2.46005 & $4.48 \times 10^{-4}$ & $1.2 \times 10^{-4}$ & $1.2 \times 10^{-4}$ \\
\hline 1 & 2.71828 & 2.71828 & 0 & 2.71828 & $4.44 \times 10^{-16}$ & $2.0 \times 10^{-9}$ & $2.0 \times 10^{-9}$ \\
\hline
\end{tabular}

TABle 11: Comparison of first-order absolute error of LSHPM with second-order HPM and VIM in Problem 11.

\begin{tabular}{lcccccc}
\hline$x$ & $\begin{array}{c}\text { Exact } \\
\text { Solution }\end{array}$ & Solution & LSHPM & & HPM & VIM \\
& 1 & 1 & Error & Solution & 0 & Error \\
0 & 0.994654 & 0.994654 & $5.55 \times 10^{-16}$ & 0.994654 & $1.32 \times 10^{-13}$ & $1.22 \times 10^{-15}$ \\
0.1 & 0.977122 & 0.977122 & $1.33 \times 10^{-15}$ & 0.977122 & $1.55 \times 10^{-12}$ & $4.44 \times 10^{-16}$ \\
0.2 & 0.944901 & 0.944901 & $2.66 \times 10^{-15}$ & 0.944901 & $5.52 \times 10^{-12}$ & $9.99 \times 10^{-16}$ \\
0.3 & 0.895095 & 0.895095 & $4.99 \times 10^{-15}$ & 0.895095 & $1.14 \times 10^{-11}$ & $4.55 \times 10^{-15}$ \\
0.4 & 0.824361 & 0.824361 & $8.65 \times 10^{-15}$ & 0.824361 & $1.67 \times 10^{-11}$ & $7.32 \times 10^{-15}$ \\
0.5 & 0.728848 & 0.728848 & $4.88 \times 10^{-15}$ & 0.728848 & $1.83 \times 10^{-11}$ & $1.02 \times 10^{-14}$ \\
0.6 & 0.604126 & 0.604126 & $3.10 \times 10^{-15}$ & 0.604126 & $1.46 \times 10^{-11}$ & $1.22 \times 10^{-14}$ \\
0.7 & 0.445108 & 0.445108 & $2.10 \times 10^{-15}$ & 0.445108 & $7.56 \times 10^{-12}$ & $1.50 \times 10^{-14}$ \\
0.8 & 0.24596 & 0.24596 & $1.30 \times 10^{-15}$ & 0.24596 & $1.52 \times 10^{-12}$ & $1.06 \times 10^{-14}$ \\
0.9 & 0 & $-2.02 \times 10^{-16}$ & $2.02 \times 10^{-16}$ & 0 & 0 & $1.17 \times 10^{-14}$ \\
1 & & & & & & 0 \\
\hline
\end{tabular}

TABLE 12: Comparison of zeroth-order absolute error of LSHPM with HPM in Problem 12.

\begin{tabular}{|c|c|c|c|c|c|}
\hline \multirow{2}{*}{$x$} & \multirow{2}{*}{$\begin{array}{c}\text { Exact } \\
\text { Solution }\end{array}$} & \multicolumn{2}{|c|}{ LSHPM } & \multicolumn{2}{|c|}{ HPM } \\
\hline & & Solution & Error & Solution & Error \\
\hline 0 & 1 & 1 & 0 & 1 & 0 \\
\hline 0.1 & 1.10517 & 1.10517 & $4.76 \times 10^{-9}$ & 1.10517 & $4.11 \times 10^{-8}$ \\
\hline 0.2 & 1.2214 & 1.2214 & $5.04 \times 10^{-8}$ & 1.2214 & $5.42 \times 10^{-7}$ \\
\hline 0.3 & 1.34986 & 1.34986 & $1.60 \times 10^{-7}$ & 1.34986 & $2.28 \times 10^{-6}$ \\
\hline 0.4 & 1.49182 & 1.49182 & $2.99 \times 10^{-7}$ & 1.49183 & $6.12 \times 10^{-6}$ \\
\hline 0.5 & 1.64872 & 1.64872 & $3.95 \times 10^{-7}$ & 1.64873 & $1.30 \times 10^{-5}$ \\
\hline 0.6 & 1.82212 & 1.82212 & $3.90 \times 10^{-7}$ & 1.82214 & $2.46 \times 10^{-5}$ \\
\hline 0.7 & 2.01375 & 2.01375 & $2.83 \times 10^{-7}$ & 2.0138 & $4.33 \times 10^{-5}$ \\
\hline 0.8 & 2.22554 & 2.22554 & $1.32 \times 10^{-7}$ & 2.22561 & $7.28 \times 10^{-5}$ \\
\hline 0.9 & 2.4596 & 2.4596 & $2.44 \times 10^{-8}$ & 2.45972 & $1.18 \times 10^{-4}$ \\
\hline 1 & 2.71828 & 2.71828 & 0 & 2.71847 & $1.84 \times 10^{-4}$ \\
\hline
\end{tabular}

$$
\begin{aligned}
\widetilde{G}(x)= & 12.4982-11.4982 e^{x}+11.4982 x+5.24912 x^{2}+1.58304 x^{3}+0.354093 x^{4} \\
& +0.0624853 x^{5}+0.00902533 x^{6}+0.00109092 x^{7}+0.000111561 x^{8} \\
& +9.64481 \times 10^{-6} x^{9}+6.83273 \times 10^{-7} x^{10}+4.08283 \times 10^{-8} x^{11}
\end{aligned}
$$




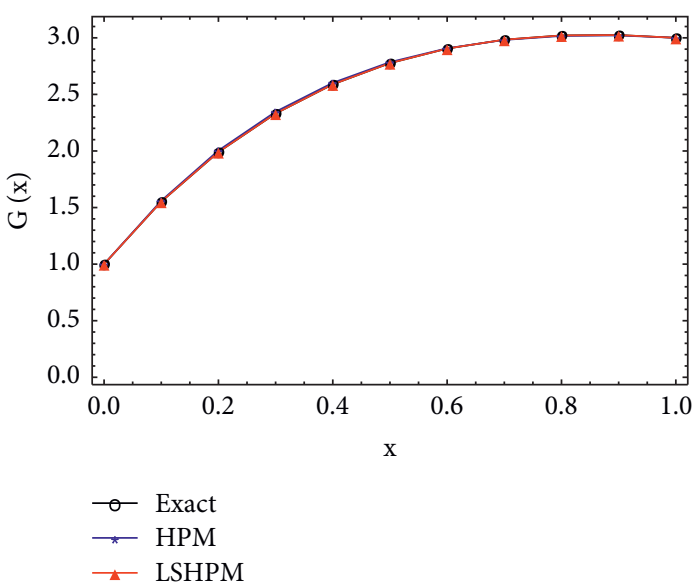

(a)

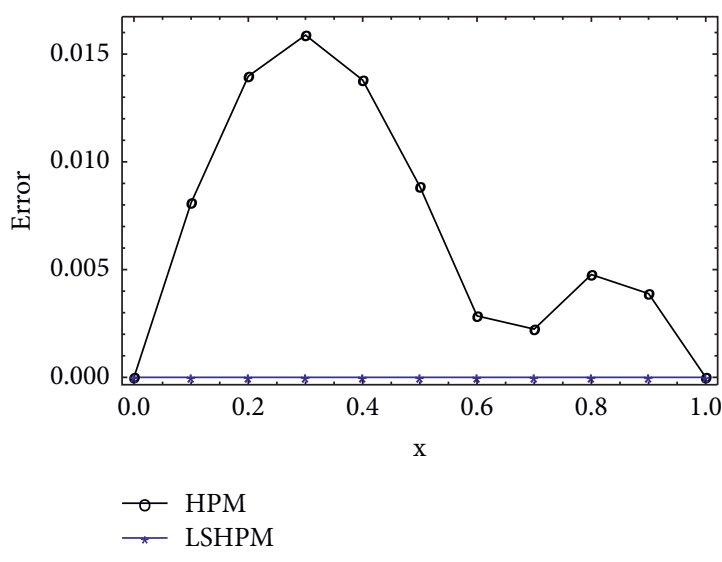

(b)

FIGURE 1: Graphical representation of solutions and errors in Problem 1. (a) Comparison of Exact, HPM, and LSHPM solutions. (b) Comparison of absolute errors of HPM and LSHPM.

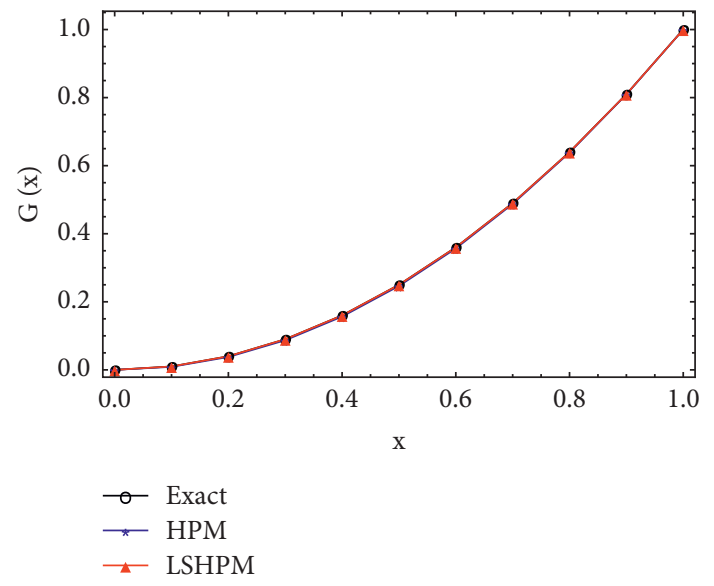

(a)

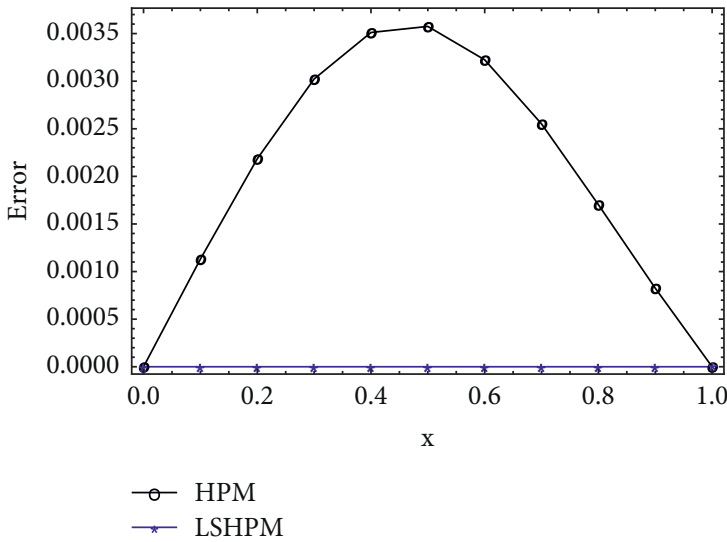

(b)

Figure 2: Graphical representation of solutions and errors in Problem 2. (a) Comparison of Exact, HPM, and LSHPM Solutions. (b) Comparison of absolute errors of HPM and LSHPM.

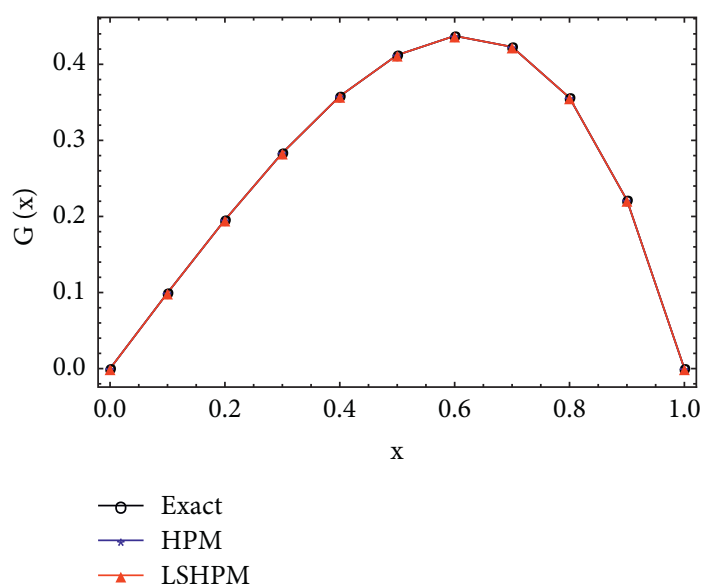

(a)

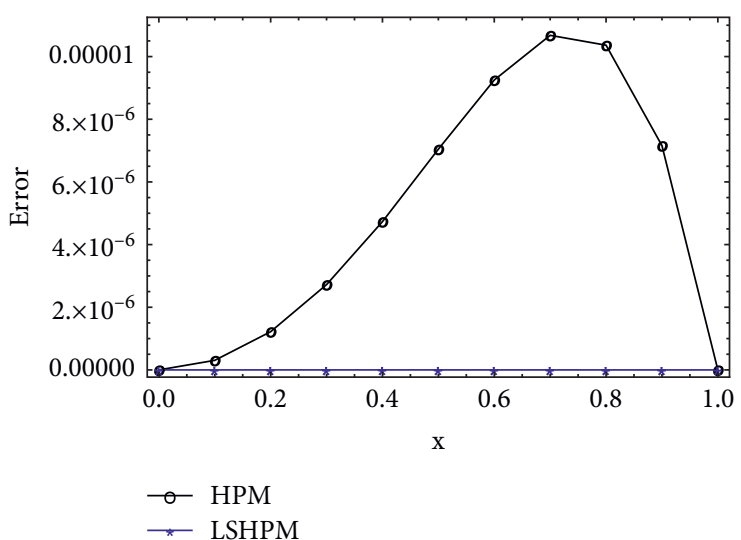

(b)

Figure 3: Graphical representation of solutions and errors in Problem 3. (a) Comparison of Exact, HPM, and LSHPM Solutions. (b) Comparison of absolute errors of HPM and LSHPM. 


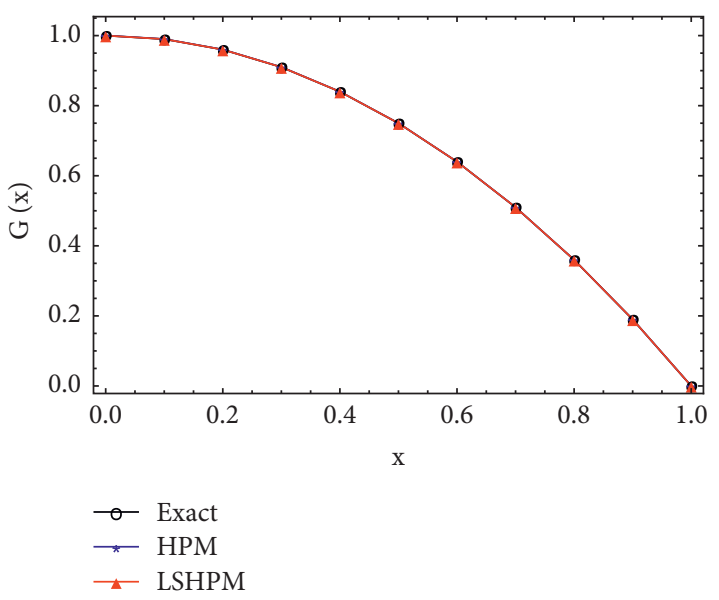

(a)

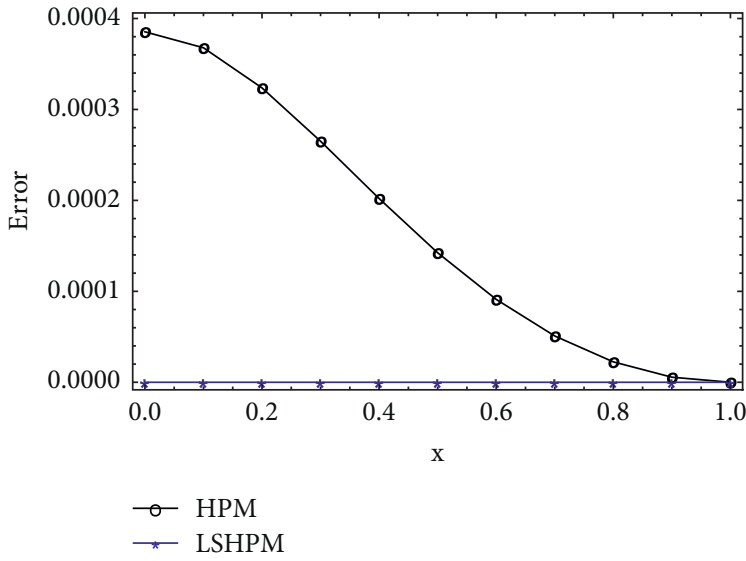

(b)

Figure 4: Graphical representation of solutions and errors in Problem 4. (a) Comparison of Exact, HPM, and LSHPM solutions. (b) Comparison of absolute errors of HPM and LSHPM.

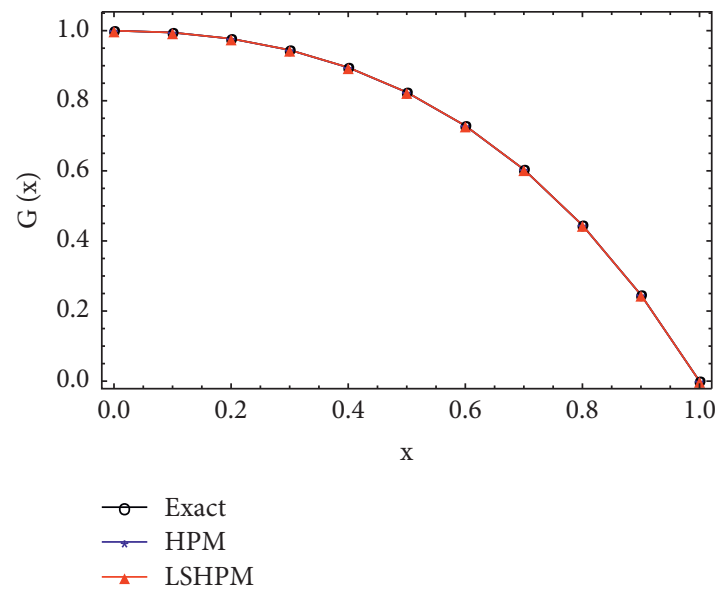

(a)

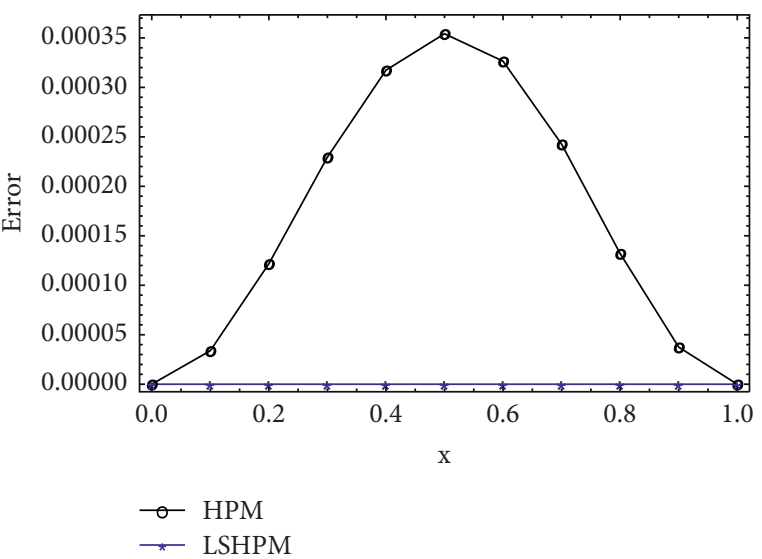

(b)

Figure 5: Graphical representation of solutions and errors in Problem 5. (a) Comparison of Exact, HPM, and LSHPM solutions. (b) Comparison of absolute errors of HPM and LSHPM.

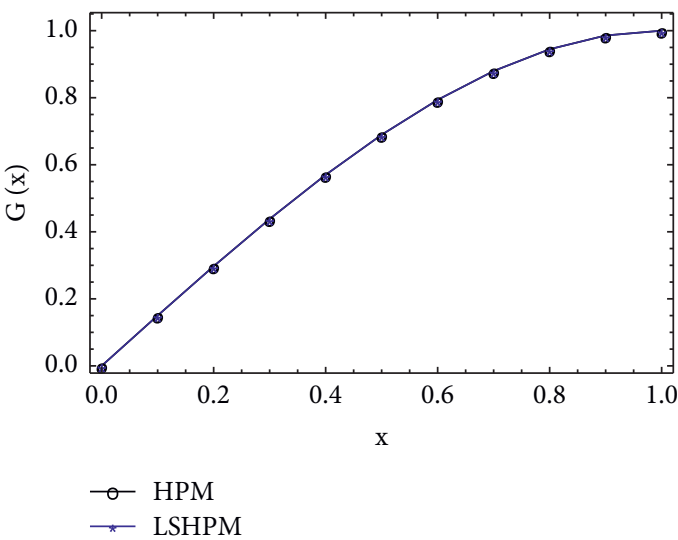

(a)

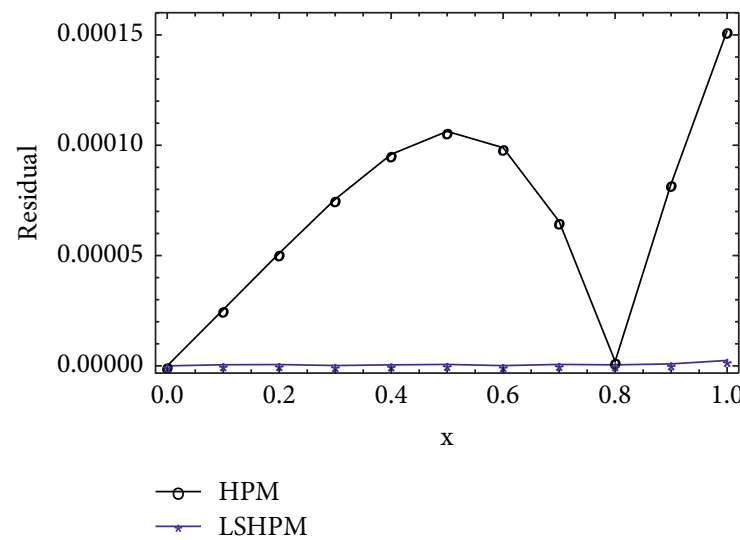

(b)

FIGURE 6: Graphical representation of solutions and errors in Problem 6. (a) Comparison of HPM and LSHPM solutions. (b) Comparison of absolute residual errors of HPM and LSHPM. 


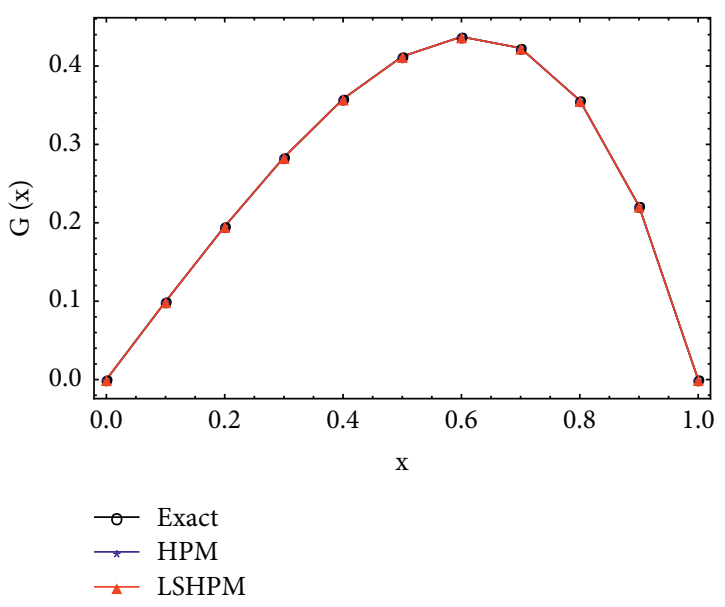

(a)

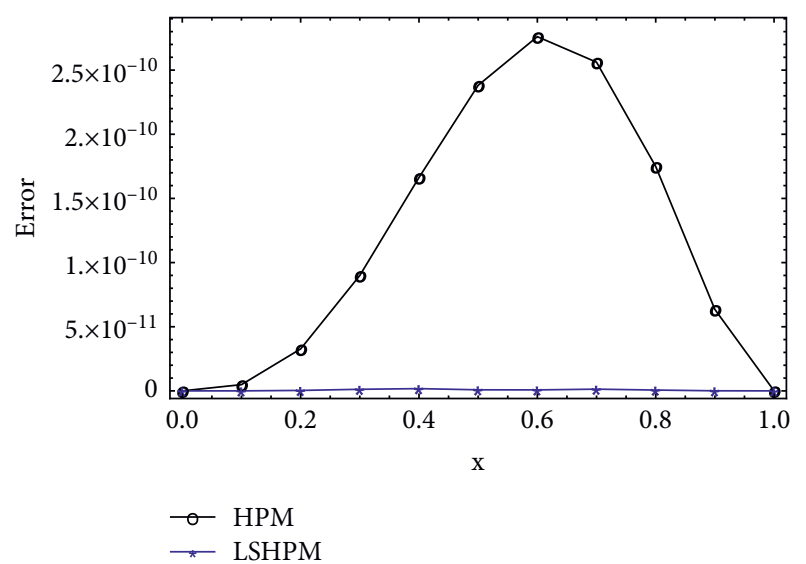

(b)

FIGURE 7: Graphical representation of solutions and errors in Problem 7. (a) Comparison of Exact, HPM, and LSHPM solutions. (b) Comparison of absolute errors of HPM and LSHPM.

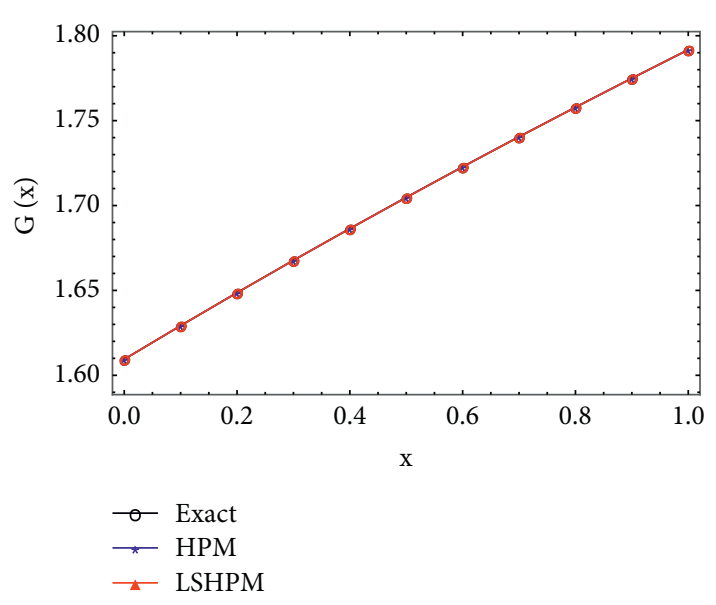

(a)

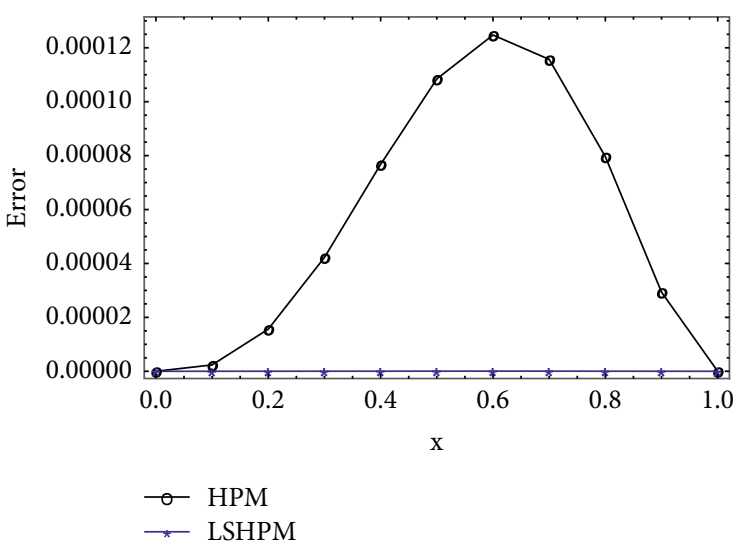

(b)

FIGURE 8: Graphical representation of solutions and errors in Problem 8. (a) Comparison of Exact, HPM, and LSHPM solutions. (b) Comparison of absolute errors of HPM and LSHPM.

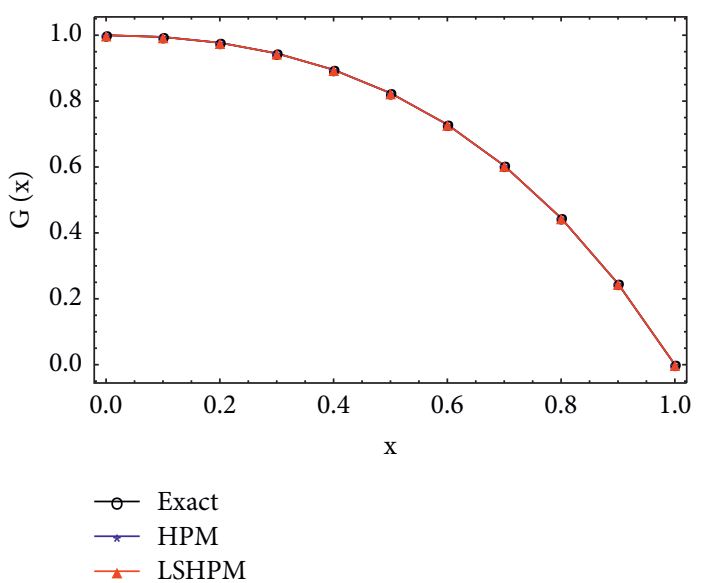

(a)

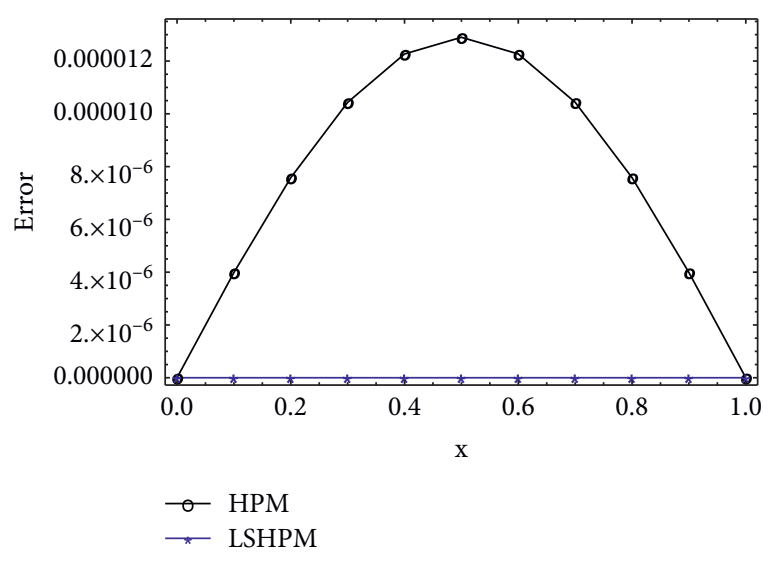

(b)

Figure 9: Graphical representation of solutions and errors in Problem 9. (a) Comparison of Exact, HPM, and LSHPM solutions. (b) Comparison of absolute errors of HPM and LSHPM. 


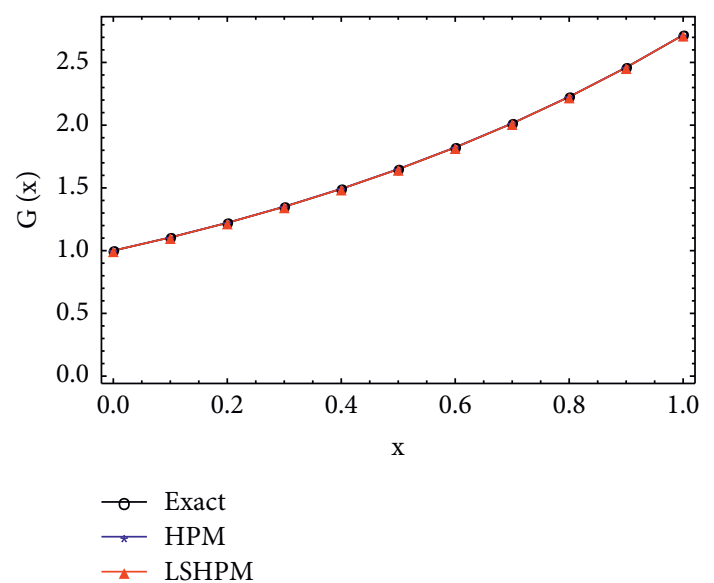

(a)

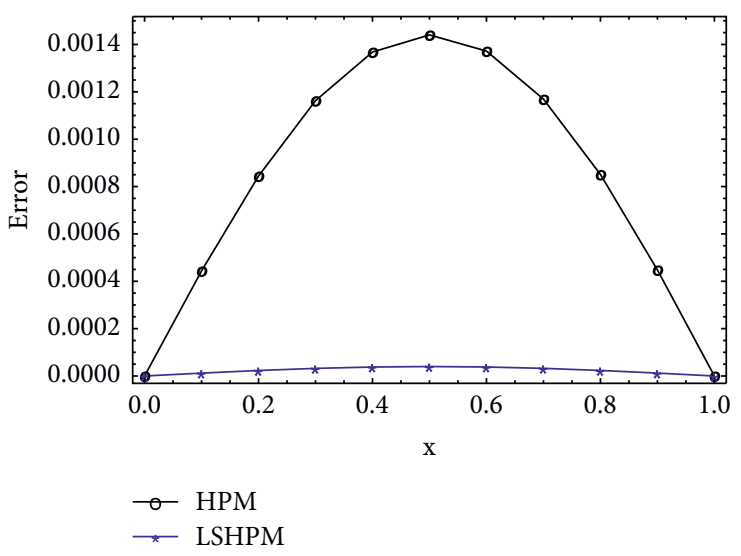

(b)

FIGURE 10: Graphical representation of solutions and errors in Problem 10. (a) Comparison of Exact, HPM, and LSHPM solutions. (b) Comparison of absolute errors of HPM and LSHPM.

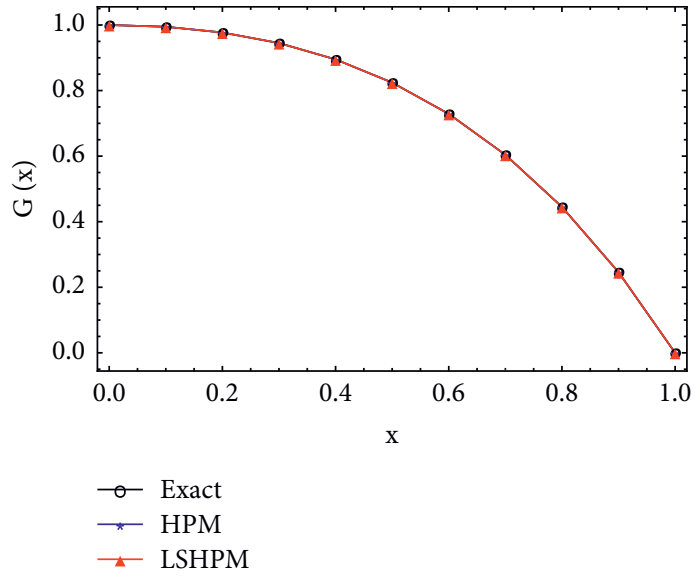

(a)

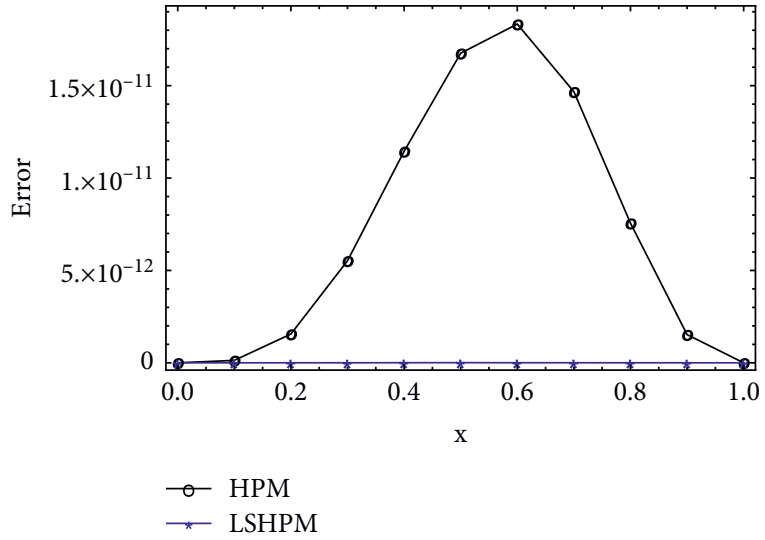

(b)

Figure 11: Graphical representation of solutions and errors in Problem 11. (a) Comparison of Exact, HPM, and LSHPM solutions. (b) Comparison of absolute errors of HPM and LSHPM.

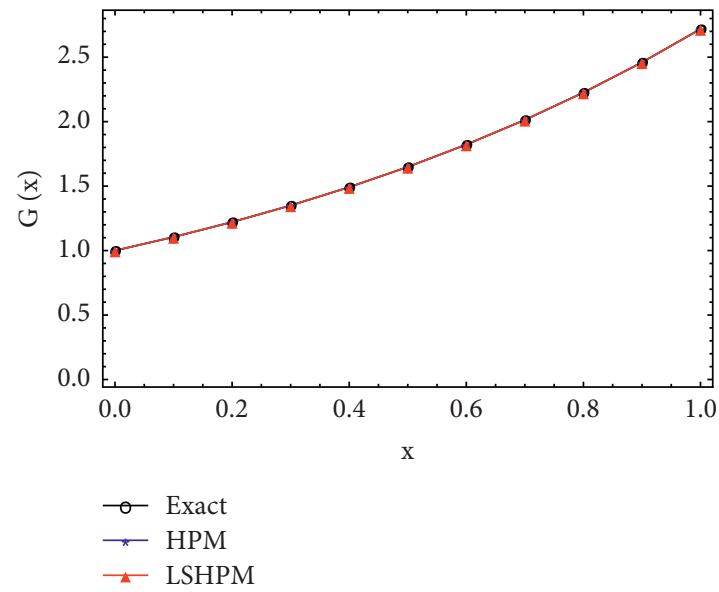

(a)

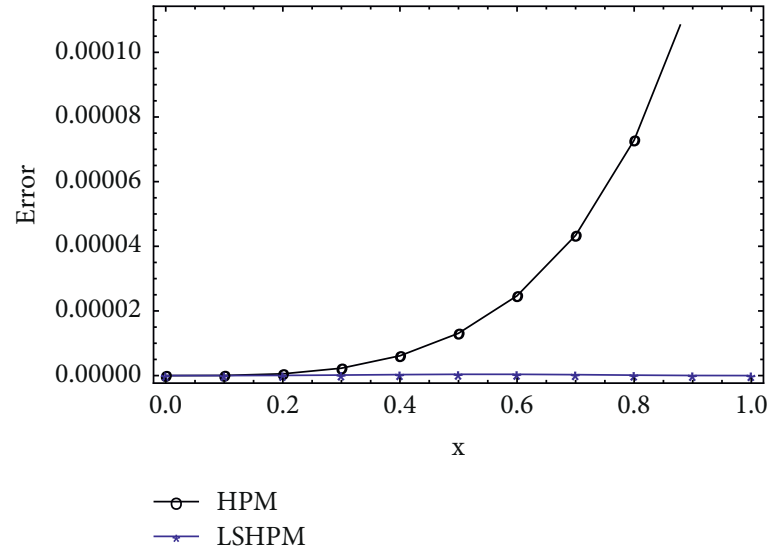

(b)

Figure 12: Graphical representation of solutions and errors in Problem 12. (a) Comparison of Exact, HPM, and LSHPM solutions. (b) Comparison of absolute errors of HPM and LSHPM. 
The results are presented in Table 9.

Problem 10. Sixth-order nonlinear ODE [14]:

$$
G^{(v i)}(x)-e^{-x} G^{2}(x)=0, \quad 0<x<1,
$$

subject to boundary conditions

$$
\begin{aligned}
G(0) & =1, \\
G^{\prime \prime}(0) & =1, \\
G^{i v}(0) & =1,
\end{aligned}
$$

$$
\begin{aligned}
G(1) & =e, \\
G^{\prime \prime}(1) & =e, \\
G^{i v}(1) & =e .
\end{aligned}
$$

The exact solution to this problem is $e^{x}$. The zeroth-order approximate solution using LSHPM is

$$
\begin{aligned}
\widetilde{G}(x)= & 1+0.999876 x+0.5 x^{2}+0.166857 x^{3}+0.0416667 x^{4}+0.00833376 x^{5} \\
& +0.00121254 x^{6}+0.000335379 x^{7}
\end{aligned}
$$

The results are presented in Table 10 .

Problem 11. Seventh-order linear ODE [32]:

$$
G^{(v i i)}(x)-G(x)+7 e^{x}=0, \quad 0<x<1,
$$

subject to boundary conditions

$$
\begin{aligned}
G(0) & =1, \\
G^{\prime}(0) & =0, \\
G^{\prime \prime}(0) & =-1,
\end{aligned}
$$

$$
\begin{gathered}
G^{\prime \prime \prime}(0)=-2, \\
G(1)=0, \\
G^{\prime}(1)=-e, \\
G^{\prime \prime}(1)=-2 e .
\end{gathered}
$$

The exact solution to this problem is $(1-x) e^{x}$. The firstorder approximate solution using LSHPM is

$$
\begin{aligned}
\widetilde{G}(x)= & 7.99997-6.99997 e^{x}+6.99997 x+2.99998 x^{2}+0.833328 x^{3}+0.166665 x^{4} \\
& +0.0249997 x^{5}+0.00277773 x^{6}+0.000198406 x^{7}-2.75823 \times 10^{-6} x^{9} \\
& -5.4721 \times 10^{-7} x^{10}-7.9003 \times 10^{-8} x^{11}-6.14598 \times 10^{-9} x^{12}-1.47065 \times 10^{-9} x^{13} .
\end{aligned}
$$

The results are presented in Table 11.

Problem 12. Seventh-order nonlinear ODE [32]:

$$
G^{(v i i)}(x)-e^{-x} G^{2}(x)=0, \quad 0<x<1,
$$

subject to boundary conditions,

$$
\begin{aligned}
G(0) & =1, \\
G^{\prime}(0) & =1, \\
G^{\prime \prime}(0) & =1, \\
G^{\prime \prime \prime}(0) & =1, \\
G(1) & =e, \\
G^{\prime}(1) & =e, \\
G^{\prime \prime}(1) & =e .
\end{aligned}
$$

The exact solution to this problem is $e^{x}$. The zeroth-order approximate solution using LSHPM is

$$
\begin{aligned}
\widetilde{G}(x)= & 1+x+0.5 x^{2}+0.166667 x^{3}+0.041736 x^{4}+0.00808598 x^{5}+ \\
& 0.00171879 x^{6}+0.0000744048 x^{8} .
\end{aligned}
$$

The results are presented in Table 12.

\section{Results and Discussion}

In this article, a new modification of HPM has been introduced by hybriding HPM with LS optimizer. The proposed scheme has been tested agaist various order linear and nonlinear BVPs. The validity of LSHPM has been checked by comparing exact and approximate solutions. For testing the efficiency LSHPM, problems are also solved with HPM and results are compared with LSHPM. This can easily be observed in Tables 1-12. These tables signify the efficiency of LSHPM in terms of high accuracy with less computational 
cost. The convergence of LSHPM can also be observed in Figures 1-12. These figures show that LSHPM is more consistent as compared other mentioned schemes.

\section{Conclusion}

In present article, an efficient and reliable modification of HPM is introduced by mixing HPM with the LS optimizer. The proposed scheme is tested against different order linear and nonlinear BVPs. The obtained solutions are compared with HPM and other numerical schemes available in the literature. Analysis of results shows that LSHPM is more consistent in terms of accuracy with less computational cost and can be used in different areas of science and technology.

\section{Data Availability}

All the data are available within the article.

\section{Conflicts of Interest}

The authors declare that they have no conflicts of interest.

\section{References}

[1] S. Liao, "Homotopy analysis method: a new analytical technique for nonlinear problems," Communications in Nonlinear Science and Numerical Simulation, vol. 2, no. 2, pp. 95-100, 1997.

[2] O. A. Bég, M. M. Rashidi, T. A. Bég, and M. Asadi, "Homotopy analysis of transient magneto-bio-fluid dynamics of micropolar squeeze film in a porous medium: a model for magneto-bio-rheological lubrication," Journal of Mechanics in Medicine and Biology, vol. 12, no. 3, Article ID 1250051, 2012.

[3] H. Hassan and M. M. Rashidi, "An analytic solution of micropolar flow in a porous channel with mass injection using homotopy analysis method," International Journal of $\mathrm{Nu}$ merical Methods for Heat and Fluid Flow, vol. 24, no. 2, pp. 419-437, 2014.

[4] A. Basiri Parsa, M. M. Rashidi, O. Anwar Bég, and S. M. Sadri, "Semi-computational simulation of magneto-hemodynamic flow in a semi-porous channel using optimal homotopy and differential transform methods," Computers in Biology and Medicine, vol. 43, no. 9, pp. 1142-1153, 2013.

[5] J.-H. He, "Homotopy perturbation technique," Computer Methods in Applied Mechanics and Engineering, vol. 178, no. 3-4, pp. 257-262, 1999.

[6] M. Carl, "Bender. Perturbation theory," in Encyclopedia of Physical Science and Technology, R. A. Meyers, Ed., pp. 715727, Academic Press, New York, NY, USA, 3rd edition, 2003.

[7] J.-H. He and Y. O. El-Dib, "Homotopy perturbation method for fangzhu oscillator," Journal of Mathematical Chemistry, vol. 58, no. 10, pp. 2245-2253, 2020.

[8] M. Qayyum, H. Khan, and O. Khan, "Slip analysis at fluidsolid interface in mhd squeezing flow of casson fluid through porous medium," Results in physics, vol. 7, pp. 732-750, 2017.

[9] J.-H. He, Y. O. El-Dib, and A. A. Mady, "Homotopy perturbation method for the fractal toda oscillator," Fractal and Fractional, vol. 5, no. 3, p. 93, 2021.

[10] M. Ali, N. Anjum, Q. T. Ain, and J.-H. He, "Homotopy perturbation method for the attachment oscillator arising in nanotechnology," Fibers and Polymers, vol. 22, no. 6, pp. 1601-1606, 2021.
[11] M. T. Darvishi, F. Khani, S. Hamedi-Nezhad, and S.-W. Ryu, "New modification of the hpm for numerical solutions of the sine-gordon and coupled sine-gordon equations," International Journal of Computer Mathematics, vol. 87, no. 4, pp. 908-919, 2010.

[12] J. Biazar and M. Eslami, "Modified hpm for solving systems of volterra integral equations of the second kind," Journal of King Saud University Science, vol. 23, no. 1, pp. 35-39, 2011.

[13] Y. O. El-Dib, "Homotopy perturbation method with rank upgrading technique for the superior nonlinear oscillation," Mathematics and Computers in Simulation, vol. 182, pp. 555-565, 2021.

[14] M. Qayyum, H. Khan, and O. Khan, "A new and reliable modification of homotopy perturbation method," Punjab University Journal of Mathematics, vol. 48, no. 2, 2020.

[15] C. Bota and B. Căruntu, "Approximate analytical solutions of nonlinear differential equations using the least squares homotopy perturbation method," Journal of Mathematical Analysis and Applications, vol. 448, no. 1, pp. 401-408, 2017.

[16] X.-X. Li and C.-H. He, "Homotopy perturbation method coupled with the enhanced perturbation method," Journal of Low Frequency Noise, Vibration and Active Control, vol. 38, no. 3-4, pp. 1399-1403, 2018.

[17] Q.-P. Ji, J. Wang, L.-X. Lu, and C.-F. Ge, "Li-He's modified homotopy perturbation method coupled with the energy method for the dropping shock response of a tangent nonlinear packaging system," Journal of Low Frequency Noise, Vibration and Active Control, vol. 40, no. 2, pp. 675-682, 2020.

[18] N. Anjum, J.-H. He, Q. T. Ain, and D. Tian, “Li-He's modified homotopy perturbation method for doubly-clamped electrically actuated microbeams-based microelectromechanical system," Facta Universitatis-Series: Mechanical Engineering, 2021.

[19] A. J. Mohamad, "Solving second order non-linear boundary value problems by four numerical methods," Engineering and Technology Journal, vol. 28, no. 2, pp. 369-381, 2010.

[20] V. S. S. Momani, "Differential transformation method for obtaing positive solutions for two-point nonlinear boundary value problem," International Journal: Mathematical Manuscripts, vol. 1, no. 1, pp. 65-72, 2007.

[21] W. M. Abd-Elhameed and N. Anna, "A unified approach for solving linear and nonlinear odd-order two-point boundary value problems," Bulletin of the Malaysian Mathematical Sciences Society, vol. 43, no. 3, pp. 2835-2849, 2019.

[22] A. Khan and T. Aziz, "The numerical solution of third-order boundary-value problems using quintic splines," Applied Mathematics and Computation, vol. 137, no. 2-3, pp. 253-260, 2003.

[23] H. N. Caglar, S. H. Caglar, and E. H. Twizell, "The numerical solution of third-order boundary-value problems with fourthdegree B-spline functions," International Journal of Computer Mathematics, vol. 71, no. 3, pp. 373-381, 1999.

[24] Z. A. A. D. Al-Rabahi and Y. Q. Hasan, "New modified adomin decomp osition metho $\mathrm{d}$ for boundary value problems of higher-order ordinary differential equation," Asian Research Journal of Mathematics, vol. 16, no. 3, pp. 20-37, 2020.

[25] M. Aslam Noor and S. T. Mohyud-Din, "An efficient method for fourth-order boundary value problems," Computers \& Mathematics with Applications, vol. 54, no. 7-8, pp. 11011111, 2007.

[26] O. Khan, M. Qayyum, H. Khan, and M. Ali, "Improved analysis for squeezing of Newtonian material between two circular plates," Advances in Materials Science and Engineering, vol. 2017, Article ID 5703291, 9 pages, 2017. 
[27] J. Ali, S. Islam, H. Khan, and S. I. Ali Shah, "The optimal homotopy asymptotic method for the solution of higherorder boundary value problems in finite domains," Abstract and Applied Analysis, vol. 2012, Article ID 401217, 14 pages, 2012.

[28] A.-M. Wazwaz, "Approximate solutions to boundary value problems of higher order by the modified decomposition method," Computers \& Mathematics with Applications, vol. 40, no. 6-7, pp. 679-691, 2000.

[29] P. P. Kumar, "A numerical method for the solution of fifth order boundary value problem in ordinary differential equations," Vladikavkaz Mathematical Journal, vol. 19, no. 4, 2017.

[30] A.-M. Wazwaz, "The numerical solution of fifth-order boundary value problems by the decomposition method," Journal of Computational and Applied Mathematics, vol. 136, no. 1-2, pp. 259-270, 2001.

[31] M. Aslam Noor and S. T. Mohyud-Din, "A reliable approach for solving linear and nonlinear sixth-order boundary value problems," International Journal of Computational and Applied Mathematics, vol. 2, no. 2, pp. 163-173, 2007.

[32] S. S. Siddiqi, G. Akram, and M. Iftikhar, "Solution of seventh order boundary value problems by variational iteration technique," Applied Mathematical Sciences, vol. 6, no. 94, pp. 4663-4672, 2012. 